NASA Contractor Report 187139

AIAA-91-1652

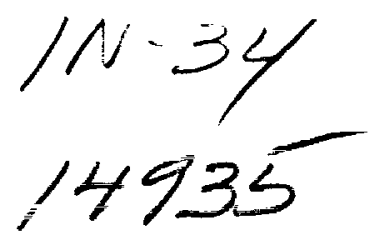

$p \cdot 1^{3}$

\title{
Time-Derivative Preconditioning for Viscous Flows
}

Yunho Choi

Sverdrup Technology, Inc.

Lewis Research Center Group

Brook Park, Ohio

and

Charles L. Merkle

The Pennsylvania State University

University Park, PA

June 1991

Prepared for

Lewis Research Center

Under Contract NAS3-25266 



\author{
Time-Derivative Preconditioning \\ for Viscous Flows \\ Yunho Choi * \\ Sverdrup Technology, Inc. \\ NASA Lewis Research Center Group \\ Brook Park, OH 44142 \\ Charles L. Merkle † \\ Department of Mechanical Engineering \\ The Pennsylvania State University \\ University Park, PA 16802
}

\begin{abstract}
A time-derivative preconditioning algorithm that is effective over a wide range of flow conditions from inviscid to very diffusive flows and from low speed to supersonic flows has been developed. This algorithm uses a "viscous" set of primary dependent variables to introduce well-conditioned eigenvalues and to avoid having a nonphysical time reversal for viscous flows. The resulting algorithm also provides a mechanism for controlling the inviscid and viscous time step parameters to be of order one for very diffusive flows, thereby ensuring rapid convergence at very viscous flows as well as for inviscid flows. Convergence capabilities are demonstrated through computation of a wide variety of problems.
\end{abstract}

\section{Introduction}

Time-marching algorithms are widely used for the computation of compressible flows. A major advantage of these techniques is that they apply to both inviscid and viscous flows and can be used in conjunction with virtually any spatial discretization in all Reynolds number regimes. In the past two or three decades, timemarching schemes have been widely accepted and applied as the method of choice for transonic, supersonic, and hypersonic flows.

In the low subsonic Mach number regime, timemarching algorithms do not fare as well. When the flow velocity becomes small in comparison with the acoustic speed, the time-dependent equations become stiff and time-marching methods converge very slowly. These difficulties are aggravated if the Reynolds number also becomes small. Many problems, however, contain some

\section{* Research Engineer}

$\dagger$ Professor

Copyright (C)1991 by the authors. Published by the American Institute of Aeronautics and Astronautics, Inc. with permission. regions with low Mach numbers while other regions are decidedly compressible so that the compressible equations have to be used throughout. Consequently, one must deal with the stiffness of the equations. Representative problems that contain both compressibility effects and low-speed regions can be grouped into two classes: high-speed flows with embedded regions of low velocity; and low-speed flows with temperature differences arising from strong heat addition. Examples of such flows are described below.

High-speed flows with embedded regions of low velocity are typified by external, transonic flow with embedded low-speed regions near stagnation points or by internal flows with low velocity flow upstream of a choked area. These embedded regions have little effect on convergence when the low-speed region is small, but they can dominate convergence when the region is large. For example, the low-speed region near the stagnation point of an isolated airfoil is seldom large enough to affect convergence, but the subsonic flow upstream of a strongly converging nozzle may control the convergence process.

Low-speed flows that are compressible because of density changes induced by heat addition can be represented by problems with surface heat transfer or volumetric heat addition. The most common example of volumetric heat addition occurs in combustion problems, but additional problems of interest include advanced space propulsion concepts such as laser, solar, and microwave thermal propulsion [1] in which electromagnetic radiation is used to heat a flowing gas. In problems such as these, the equations frequently remain stiff over the entire computational domain, so that efficiency requirements make it imperative that the stiffness be dealt with directly.

Several previous researchers [2-12] have considered the low Mach number problem for inviscid flows. The present paper is directed towards viscous flows.

\section{Previous Work on Eigenvalue Control}

Two distinct methods have been suggested for con- 
trolling eigenvalue stiffness at low speeds to enhance convergence. The first is to premultiply the time derivative by a suitable matrix that scales the eigenvalues of the system to the same order of magnitude. The other is to use a perturbed form of the equations in which the physical acoustic waves are replaced by pseudo-acoustic modes. We refer to the former as preconditioning and to the latter as a perturbation procedure. The preconditioning method has the advantage that it provides a global solution that is valid at all Mach numbers, whereas the perturbation method is valid only locally in the regime in which the perturbation is carried out.

A number of preconditioning studies have been reported previously. Viviand [2] considered generalized preconditioning procedures for a class of hyperbolic systems representing the Euler equations and gives specific rules that ensure that the preconditioned equations remain well posed. Additional details concerning preconditioning are presented by Peyret and Viviand [3], who are the only ones so far to consider preconditioning in viscous flows. Turkel [4] also discusses preconditioning with applications to both compressible and incompressible flows. Finally, Storti et al. [5] have presented some recent theoretical work on eigenvalue control that agrees well with Viviand's findings. References 2 through 5 are primarily theoretical in nature and consider the generalized concept of preconditioning in any computational regime, not just the low Mach number regime with which we are here concerned. Numerical studies of preconditioning in the low Mach number regime have been reported by Briley et al. [6] for isoenergetic systems and by the present authors and their coworkers [7-9] for the Euler equations. Low Mach number convergence enhancement by Briley et al. was limited to Mach numbers above 0.05 . Our results demonstrated that preconditioning provided Mach number independent convergence at all Mach numbers between 0.7 and $10^{-6}$. In addition some applications of "time inclining" have been reported by Dannenhoffer and Giles [10]. All of these previous works have considered the inviscid equations.

The second general method for eliminating eigenvalue stiffness is to use a perturbed form of the equations of motion. The present authors $[8,11]$ have used an expansion of the flow variables in terms of the Mach number squared to remove the physical acoustic waves and replace them by a set of pseudo-acoustic waves whose speeds are comparable to the particle velocity. A similar perturbation procedure has also been developed by Guerra and Gustafsson [12] based upon expansion in Mach number. The perturbation method is effective for both viscous and inviscid flows and has been implemented for numerous applications [13-15]. Other perturbation procedures include the one by Rehm and
Baum [16], which is specialized toward combustion problems and has been applied by several authors including Chenoweth and Paolucci [17] and McMurtry et al. [18]. Although these perturbation procedures are robust, the nature of the perturbation limits their usage to low subsonic flows. Specifically, the methods are not adequate for the transonic flow regime. Our focus here is on the preconditioning methods, but we take advantage of knowledge gained from perturbation methods to develop the preconditioning matrix.

To date, applications of preconditioning to viscous flows have not been reported. We attempted several numerical studies for viscous flows using the preconditioning method of $[7,9]$ and found that it is not effective in viscous flows. Accordingly, the purpose of the present paper is to develop a method which gives reasonable convergence for all Reynolds number ranges while keeping its effectiveness in inviscid flows as in the previous method.

\section{Problem Formulation}

\subsection{Equations of Motion}

The two-dimensional compressible Navier-Stokes equations using time-derivative preconditioning can be written in the following vector form :

$$
\Gamma \frac{\partial Q}{\partial t}+\frac{\partial E}{\partial x}+\frac{\partial F}{\partial y}=H+L\left(Q_{v}\right)
$$

where $\Gamma$ is the preconditioning matrix and will take on various forms depending on the preconditioning chosen. When $\Gamma$ is the identity matrix, we recover the standard (nonpreconditioned) equations. The vectors in Eq.(1) are

$$
\begin{aligned}
Q & =(\rho, \rho u, \rho v, e)^{T} \\
E & =\left(\rho u, \rho u^{2}+p, \rho u v,(e+p) u\right)^{T} \\
F & =\left(\rho v, \rho u v, \rho v^{2}+p,(e+p) v\right)^{T} \\
H & =(0,0,-\rho g, q-\rho g v)^{T} \\
Q_{v} & =(p, u, v, T)^{T}
\end{aligned}
$$

In these expressions, the dependent vector $Q$ and the inviscid flux vectors $E$ and $F$ take their standard form, and $H$ is the source vector, which contains both a volumetric heat addition rate and a gravitational body force. The vector $Q_{v}$ represents the "viscous" variables that appear in the diffusion operators.

The variables in Eq.(2) are defined by standard notation including density $\rho$, velocity components $u$ and $v$, pressure $p$, temperature $T$, Mach number $M$ and total energy per unit volume $e$. The coordinate system is oriented so that the gravitational body force is in the 
negative $y$-direction. Heat is added to the flow by specifying the form of the volumetric heat addition function q. The gravitational term $\rho g v$ is the work done by the gravity force. The pressure obtained from the perfect gas relation is

$$
p=(\gamma-1)\left(e-\frac{\rho}{2}\left(u^{2}+v^{2}\right)\right)
$$

where $\gamma$ is the ratio of specific heats.

The differential operator for the viscous terms $\mathrm{L}$ is

$$
L=\frac{\partial}{\partial x} R_{x x} \frac{\partial}{\partial x}+\frac{\partial}{\partial x} R_{x y} \frac{\partial}{\partial y}+\frac{\partial}{\partial y} R_{y x} \frac{\partial}{\partial x}+\frac{\partial}{\partial y} R_{y y} \frac{\partial}{\partial y}
$$

The matrices $R_{x x}, R_{x y}, R_{y x}$, and $R_{y y}$ are diffusion coefficient matrices that include the viscosity $\mu$ and the thermal conductivity $\mathbf{k}$ :

$R_{x x}=\left(\begin{array}{cccc}0 & 0 & 0 & 0 \\ 0 & \frac{4}{3} \mu & 0 & 0 \\ 0 & 0 & \mu & 0 \\ 0 & \frac{4}{3} \mu u & \mu v & k\end{array}\right) \quad R_{x y}=\left(\begin{array}{cccc}0 & 0 & 0 & 0 \\ 0 & 0 & -\frac{2}{3} \mu & 0 \\ 0 & \mu & 0 & 0 \\ 0 & \mu v & -\frac{2}{3} \mu u & 0\end{array}\right)$

$R_{y x}=\left(\begin{array}{cccc}0 & 0 & 0 & 0 \\ 0 & 0 & \mu & 0 \\ 0 & -\frac{2}{3} \mu & 0 & 0 \\ 0 & -\frac{2}{3} \mu v & \mu u & 0\end{array}\right) \quad R_{y y}=\left(\begin{array}{cccc}0 & 0 & 0 & 0 \\ 0 & \mu & 0 & 0 \\ 0 & 0 & \frac{4}{3} \mu & 0 \\ 0 & \mu u & \frac{4}{3} \mu v & k\end{array}\right)$

In these expressions, Stoke's hypothesis is used for the second coefficient of viscosity $\left(\lambda=-\frac{2}{3} \mu\right)$.

\subsection{Development of Preconditioning Procedure}

The preconditioning matrix that was chosen in our earlier work $[7,9]$ left the continuity and momentum equations in their traditional form but modified the energy equation such that time derivatives of $\rho, \rho u$, and $\rho v$ were added. Both stability analyses and numerical experiments showed that this preconditioning matrix was effective for a wide variety of low Mach number inviscid calculations [7,9]. Stability analysis of the full Navier-Stokes equations, however, as well as numerical experiments shows it is unstable at low Reynolds numbers. Detailed analysis [8] indicates that this instability depends primarily upon the Prandtl number. This dependence suggests that the modified energy equation may no longer be well posed in the diffusion limit. To circumvent this difficulty, a new preconditioning matrix that is effective both in inviscid and viscous calculations is developed in the present paper.

The basic idea of the new time-derivative preconditioning procedure is to employ a "viscous" set of dependent variables $\left(Q_{v}\right)$ as the primary dependent vector. This set of variables is inspired by artificial compressibility methods for incompressible flow $[4,7]$ as well as low Mach number formulations using perturbation expansions for compressible flow $[8,11]$. The low Mach number formulation with this set of variables proves to give unconditionally stable results for both inviscid and viscous calculations.

In order to simplify the algebra, first we transform the conservative form of Eq.(1) to the non-conservative form. We define the non-conservative vector as

$$
\tilde{Q}=(\rho, u, v, p)^{T}
$$

where the tilde represents the non-conservative variable. We then premultiply Eq.(1) by the Jacobian $P^{-1}$ $=\partial \tilde{Q} / \partial Q$ to obtain

$$
\frac{\partial \tilde{Q}}{\partial t}+P^{-1}\left(\frac{\partial E}{\partial x}+\frac{\partial F}{\partial y}\right)=P^{-1}\left(H+L\left(Q_{v}\right)\right)
$$

Here the preconditioning matrix is dropped for the time being.

One convenient method for obtaining a new preconditioning matrix, as well as for transforming the $(\rho, u, v, p)$ system to the $(\mathrm{p}, \mathrm{u}, \mathrm{v}, \mathrm{T})$ system, is as follows. First we subtract the continuity equation from the energy equation. This introduces the temperature form of the time derivative into the energy equation $(\partial \rho / \partial t$ in the continuity equation is replaced by $\partial T / \partial t$ by means of the perfect gas law.) This temperature form of the time derivative also appears well-suited to the heat diffusion term. The vector form of this step is obtained by premultiplying Eq.(7) by a matrix $K_{1}$.

$$
K_{1} \frac{\partial \tilde{Q}}{\partial t}+K_{1} P^{-1}\left(\frac{\partial E}{\partial x}+\frac{\partial F}{\partial y}\right)=K_{1} P^{-1}\left(H+L\left(Q_{v}\right)\right)
$$

where the sparse matrix $K_{1}$ is

$$
\begin{aligned}
& K_{1}=\operatorname{Diag}(1, \rho, \rho, 1), \\
& \text { with nonzero element }(4,1)=-\gamma R T
\end{aligned}
$$

We then convert the dependent vector $\tilde{Q}$ to $Q_{v}$ by using the chain rule,

$$
K_{1} K_{2} \frac{\partial Q_{v}}{\partial t}+K_{1} P^{-1}\left(\frac{\partial E}{\partial x}+\frac{\partial F}{\partial y}\right)=K_{1} P^{-1}\left(H+L\left(Q_{v}\right)\right)
$$

where $K_{2}$ is defined as the Jacobian, $K_{2}=\partial \tilde{Q} / \partial Q_{v}$. Now we precondition Eq.(10) by premultiplying the time derivative term by $\Gamma_{v} K_{2}^{-1} K_{1}^{-1}$ to get

$$
\Gamma_{v} \frac{\partial Q_{v}}{\partial t}+K_{1} P^{-1}\left(\frac{\partial E}{\partial x}+\frac{\partial F}{\partial y}\right)=K_{1} P^{-1}\left(H+L\left(Q_{v}\right)\right)
$$


where the preconditioning matrix $\Gamma_{v}$ is defined as

$$
\Gamma_{v}=\left(\begin{array}{cccc}
\frac{1}{\beta M^{2}} & 0 & 0 & 0 \\
0 & \rho & 0 & 0 \\
0 & 0 & \rho & 0 \\
(1-\gamma) & 0 & 0 & \gamma \rho R
\end{array}\right)
$$

This choice of the preconditioning matrix $\Gamma_{v}$ introduces the artificial compressibility formulation of the continuity equation and modifies the energy equation to its classical temperature form, while keeping momentum equations in their standard form. These equations are also nearly identical to the perturbation equations used extensively $[8,11,13-15]$. Here, $\beta M^{2}$ is a scaling parameter ( $M$ is Mach number), the proper choice of which is discussed later. Note that the momentum and energy equations now appear to be well-suited for viscous effects, since they are all of the form

$$
\frac{\partial \phi}{\partial t}+V \cdot \nabla \phi=\nabla^{2} \phi
$$

where $\phi$ represents $u, v$, and $T$, respectively.

The corresponding time-derivative preconditioned system for the conservative form of the equations is readily found by premultiplying Eq.(11) by $P K_{1}^{-1}$.

$$
\bar{\Gamma} \frac{\partial Q_{v}}{\partial t}+\frac{\partial E}{\partial x}+\frac{\partial F}{\partial y}=H+L\left(Q_{v}\right)
$$

Here $\bar{\Gamma}$, defined as $\bar{\Gamma}=P K_{1}^{-1} \Gamma_{v}$, is

$$
\bar{\Gamma}=\left(\begin{array}{cccc}
\frac{1}{\beta M^{2}} & 0 & 0 & 0 \\
\frac{y}{\beta M^{2}} & \rho & 0 & 0 \\
\frac{v}{\beta M^{2}} & 0 & \rho & 0 \\
\frac{(c+p)}{\rho \beta M^{2}}-1 & \rho u & \rho v & \frac{\gamma \rho R}{\gamma-1}
\end{array}\right)
$$

The eigenvalues of the preconditioned system of equations (14) are

$$
\lambda\left(\bar{\Gamma}^{-1} A_{v}\right)=\left(u, u, \frac{u\left(1+\frac{\beta M^{2}}{\gamma R T}\right) \pm c^{\prime}}{2}\right)
$$

where $A_{v}=\partial E / \partial Q_{v}$ and the pseudo-acoustic speed $c^{\prime}$ is defined as

$$
c^{\prime 2}=u^{2}\left(1-\frac{\beta M^{2}}{\gamma R T}\right)^{2}+4 \beta M^{2}
$$

In order to get well-conditioned eigenvalues for the inviscid case, we choose the scaling parameter $\beta=\mathrm{k} \gamma R T$, where $\mathrm{k}$ is a parameter to be determined later. For values of $k$ of order unity, this choice of $\beta$ allows the pseudo-acoustic wave speed $c^{\prime}$ to be the same order as the particle velocity $u$ and ensures that three eigenvalues are always positive, while one is negative for subsonic flow.

\subsection{Convergence Control at Low Reynolds Numbers}

The stability analysis of Eq.(14) shows that the new preconditioning algorithm provides appropriate stability for all Reynolds and Peclet numbers. This new preconditioning algorithm appears to be at least as good as the original preconditioning algorithm for inviscid flow and is a dramatic improvement over the original one for viscous flows. At low Reynolds number flow (below Re $=500$ ), however, stability characteristics show that amplification factors approach unity, suggesting slow convergence rates. The reason for this behavior can be understood by considering the viscous time step parameter (hereinafter referred to as the von Neumann number) $\sigma=\mu \Delta t / \rho \Delta x^{2}$, which becomes important at low Reynolds numbers. Control of the CFL number alone at low Reynolds number makes the von Neumann number so large that the approximate factorization error in the diffusive terms slows convergence. For efficient convergence, we should control both the CFL number and the von Neumann number to be order of unity simultaneously.

In the present paper, the simultaneous control of both the CFL number and the von Neumann number is obtained by choosing the scaling parameter $\mathrm{k}$ in the definition $\beta$. The parameter $k$ is chosen as unity ( $\beta=$ $\gamma R T$ ) to get well-conditioned eigenvalues for the inviscid terms. In low Reynolds number flow, however, we use this parameter to specify both CFL and von Neumann numbers. In the inviscid limit, we choose the time step by an appropriate CFL number,

$$
C F L=\frac{\left(u\left(1+k M^{2}\right)+c^{\prime}\right) \Delta t}{2 \Delta x}
$$

while in the viscous limit the appropriate viscous time step parameter is the von Neumann number. By solving Eq.(18) and the above von Neumann number definition for $\mathrm{k}$, we find

$$
k=\frac{\alpha(\alpha-1)}{M^{2}\left(\alpha-1+\frac{\gamma R T}{u^{2}}\right)}
$$

where $\alpha$ is CFL/ $\sigma e_{\Delta x}$ and $R e_{\Delta x}$ is the cell Reynolds number $(=\rho u \Delta x / \mu)$. Now, by setting the CFL number and the von Neumann number to order unity and computing other variables from given conditions, we can obtain the scaling factor $\mathbf{k}$. In general, for a wide range of Reynolds number, the parameter $k$ can be expressed as

$$
k=\operatorname{Max}\left[1, \frac{\alpha(\alpha-1)}{M^{2}\left(\alpha-1+\frac{\gamma R T}{u^{2}}\right)}\right]
$$




\section{Numerical Solution Procedure}

Appropriate preconditioning enhances convergence of either explicit or implicit algorithms [9]. Here, the numerical solution of Eq.(14) is obtained by using an Euler implicit discretization in time along with central differencing in space. For the efficient solution of the resulting matrix, we use an approximate factorization such as the Douglas-Gunn procedure $[19,20]$. This leads to

$$
\begin{aligned}
& {\left[I+\Delta t S^{-1}\left(\frac{\partial A}{\partial x}-\frac{\partial}{\partial x} R_{x x} \frac{\partial}{\partial x}\right)\right]} \\
& {\left[I+\Delta t S^{-1}\left(\frac{\partial B}{\partial y}-\frac{\partial}{\partial y} R_{y y} \frac{\partial}{\partial y}\right)\right] \Delta Q_{v}=-\Delta t S^{-1} R}
\end{aligned}
$$

where $R$ is the residual of the steady state version of Eq.(14)

$$
R=\frac{\partial E}{\partial x}+\frac{\partial F}{\partial y}-L\left(Q_{v}\right)-H
$$

Here A, B and D are Jacobians of the vectors E, F, and $\mathrm{H}$ and the matrix $\mathrm{S}$ is defined as $\mathrm{S}=\bar{\Gamma}-\Delta t D$. This formulation differs from the traditional approximately factored algorithm only in the calculation of the preconditioning matrix, and hence additional computational cost is negligible.

\section{Boundary Conditions}

In the present study, Method of Characteristics based boundary conditions [11] are used at inflow and outflow boundaries. In this procedure, it is imperative to incorporate the preconditioning matrix to reflect the character of pseudo-acoustic waves. To apply the Method of Characteristics procedure, we first premultiply Eq.(21) by the modal matrix $M^{-1}$ containing the left eigenvector of the Jacobian $\Gamma^{-1} A$ (or $\Gamma^{-1} B$ ) at a constant $\mathrm{x}$ (or $\mathrm{y}$ ) boundary. We then multiply the result by a selection matrix $L$ that selects those characteristic equations that represent outgoing information at the boundaries to give

$L M^{-1}\left[I+\Delta t S^{-1} \frac{\partial A}{\partial x}\right]\left[I+\Delta t S^{-1}\left(\frac{\partial B}{\partial y}-\frac{\partial}{\partial y} R_{y y} \frac{\partial}{\partial y}\right)\right] \Delta Q_{v}$ $=-\Delta t L M^{-1} S^{-1} R$

In Eq.(23), the selection matrix $\mathrm{L}$ has different forms depending on the boundaries of interest. For inflow boundaries where the flow is subsonic, $\mathrm{L}$ becomes $L=\operatorname{Diag}(0,0,0,1)$, where the nonzero entry represents the outgoing characteristic equation $\left(u-c^{\prime}\right)$, while the zero entries require physically meaningful boundary conditions be specified. For the present study, stagnation pressure, stagnation temperature, and flow angle $(\mathrm{v} / \mathrm{u})$ are fixed.
The outflow boundary conditions are also obtained in a similar way. When flow is subsonic, a selection matrix is $\mathrm{L}=\operatorname{Diag}(1,1,1,0)$ and a constant static pressure is imposed. For supersonic flow, the selection matrix $L$ becomes the identity matrix and no boundary condition needs to be specified.

The boundary conditions imposed on the solid surface are the no-slip conditions and the normal pressure gradient condition obtained from the normal momentum equation. In addition, either a constant wall temperature or an adiabatic wall condition is specified depending on the problem. The axis of symmetry is treated as a regular field point using symmetry conditions in lieu of boundary conditions.

\section{Results}

Representative results have been obtained for various problems including flow past an isolated airfoil, flow through a converging nozzle, and flow in a thermally driven cavity. In all cases it was demonstrated that the preconditioning changed only the rate of convergence, not the final results. Consequently, we focus on comparing the convergence rates of the various problems with and without preconditioning. The saving realized with preconditioning ranges from a factor of two to several orders of magnitude.

\subsection{Flow Past an Isolated Airfoil}

The first test problem considers inviscid and viscous flow past a NACA0012 airfoil at zero angle of attack. A C-type grid (56)31) is used and the outer boundary is located 5 chord lengths away from the airfoil. Since the flowfield is symmetric, only the half domain is considered. For the wall, a slip boundary condition is used for inviscid flows, while no-slip, constant wall temperature, and zero normal pressure gradient are specified for viscous flows. At the outer boundary, a stagnation pressure, a stagnation temperature, and an inflow angle are specified at the inflow region, while a constant pressure is specified at the downstream end. The remaining conditions come from the Method of Characteristics. The outer free stream Mach number is $10^{-4}$.

Figure 1 shows the convergence characteristics using preconditioning for a wide range of flow conditions from inviscid flow to a very viscous flow of $R e=1$. The inviscid case indicates a rate of convergence of one order of magnitude per 200 iterations. Convergence without preconditioning required some 100,000 iterations for one order of convergence. For viscous cases at Reynolds numbers of 1,100 , and 1000 , it can be seen that the convergence rate is faster than that of the inviscid case by a factor of two. This convergence 
enhancement may be because physical diffusion serves to dissipate the errors in the solution more rapidly. At $\operatorname{Re}=10000$, however, a slight slowdown in convergence can be noted. This is because divergence was noted when the same CFL number $(\mathrm{CFL}=6)$ as that used in the low Reynolds number calculations was employed. In all cases, it is clear that the preconditioning procedure enables convergence at these very incompressible flow speeds with efficiencies that are competitive with those normally observed in subsonic flows.

The corresponding velocity fields for the three Reynolds number cases are shown in Fig.2. The top figures show full domain solutions, while the bottom figures show magnified results around the airfoil. At Reynolds number 1 , the flow is nearly symmetric around the airfoil, approaching Stokes flow. As Reynolds number increases, the boundary layer gets thinner and at Reynolds number of 10000 , separation is observed at $80 \%$ chord. The solution accuracy has been verified by comparing the pressure coefficient with incompressible panel method results for the inviscid case but this comparison is not presented for reasons of space.

\subsection{Flow Through a Strongly Converging Nozzle}

The second test problem concerns the flow through a converging-diverging nozzle. This test problem typifies a high-speed flow with an embedded region of low velocity in which the compressibility effect is significant due to the presence of transonic flow. Two nozzle geometries are considered with area ratios (AR) of 10 and 200. These nozzle geometries are of interest to us for applications in solar propulsion [1], which require large convergence because of the dilute nature of solar radiation.

First we present convergence characteristics without preconditioning for $A R=10$ for several Mach numbers in Fig.3. The calculations are made for inviscid flows, and the nozzle geometry is shown in Fig.4. Throughout the calculations, an H-grid $(71 \times 31)$ is used and the same CFL number is employed. The Mach numbers shown in Fig. 3 correspond to the Mach numbers at the throat region. For the $M=1.0$ case, the Mach number variation in the flowfield ranges from $\mathrm{M}=0.05$ in the upstream section to $\mathrm{M}=1.6$ at the nozzle exit, while for the $M=0.1$ case, it ranges from $M=0.01$ in the upstream section to $\mathrm{M}=0.08$ at the exit. It can be noted that from $M=0.7$ to 1.0 cases, convergence rates are nearly the same and it takes 2000 steps to reach the $10^{-8}$ level. This convergence rate is slow at least by a factor of seven, compared to the one with preconditioning shown in the same figure. The convergence rate with preconditioning can be understood as the one without preconditioning for high subsonic speed flow, and thus the convergence comparison suggests that the embedded low subsonic flow dominates the convergence process and slows the convergence rates. It is also interesting to note that for the $M=1.0$ case even though the flow passes the sonic line where an eigenvalue stiffness exists, the convergence rate is not affected and maintains the same convergence rate as the $M=0.9$ case. This suggests that small regions with the sonic value may have little effect on the convergence rates. For Mach numbers below 0.5 , they gradually start to slow as Mach number decreases, as expected.

Additional calculations for $A R=10$ and $A R=200$ for viscous flows were made. The $71 \times 31 \mathrm{grid}$ for $A R=10$ and the $100 \times 50$ grid for $A R=200$ are used, and they are highly clustered to the wall. In both cases, calculations go through the transonic region. Reynolds numbers based on the throat diameter are $6 \times 10^{6}$ for $A R=10$ and $9 \times 10^{5}$ for $A R=200$, respectively. Corresponding Mach number results using the preconditioning method are shown in Figs.4 and 5. Because of the strong nozzle convergence, the Mach numbers in the upstream section are about 0.05 (for $A R=10$ ) and 0.002 (for $A R=200$ ), respectively. Without preconditioning it is found that the solutions are not developed, particularly in the upstream section where the Mach number is low. In Fig.6, the convergence rates with and without preconditioning are compared. Without preconditioning, it takes 450 steps for $A R=10$ and 2100 steps for $A R=200$ to reduce one order of magnitude in $\Delta Q / Q$, while with preconditioning it takes 50 steps for $A R=10$ and 100 steps for $A R=200$. Thus, the preconditioning method enhances convergence rates by a factor of nine for $A R=10$ and twenty one for $A R=200$, respectively.

\subsection{Flow in a Thermally Driven Cavity}

Our final test problem considers a buoyancy-driven flow in a square enclosure. The configuration consists of two insulated horizontal walls and two vertical walls at temperatures $T_{h}$ and $T_{c}$. Even though this problem has been a classical natural convection problem for many decades, most studies are based on the incompressible formulation with the Boussinesq approximation [21], which requires a small temperature difference between the vertical walls. However, practical applications, such as furnace or nuclear reactor design, require much larger temperature differences, and compressible formulations without the Boussinesq assumption should be employed. Thus, for the present study, this problem is chosen as representative of a group of low-speed flows that are compressible because of density changes induced by surface heat transfer.

It is known that this problem exhibits complex flow features depending on the Rayleigh number $\left(\operatorname{Ra}=\rho^{2} g \beta\left(T_{h}-T_{c}\right) L^{3} C_{p} / \mu k\right)$, the aspect ratio, 
and the temperature difference parameter $\left(\epsilon=\left(T_{h}-\right.\right.$ $\left.\left.T_{c}\right) /\left(T_{h}+T_{c}\right)\right)$. Here $\beta$ is the thermal expansion coefficient and $\mathrm{L}$ is the enclosure length. For the present study, three Rayleigh number cases, $\operatorname{Ra}=10^{3}, 10^{5}$, and $10^{6}$, are considered with the temperature difference parameter $\epsilon=0.6$. The aspect ratio of this problem is one and the transport properties ( $\mu$ and $k$ ) are evaluated by using Sutherland's law. The Prandtl number based on reference transport properties is 0.7 . A $61 \times 61$ uniform grid is used for $\mathrm{Ra}=10^{3}$, and a $91 \times 91$ uniform grid is used for $\mathrm{Ra}=10^{5}$ and $10^{6}$.

Convergence rates with preconditioning for the three cases are shown in Fig.7. In all cases, the convergence behavior shows two distinct slopes. At the initial stage, convergence is fast, but it slows down after a certain step. The most prominent case is $\mathrm{Ra}=10^{3}$. At the initial convergence process, 80 steps are required for one order of magnitude drop of $\Delta Q / Q$, but after 500 steps the convergence rate becomes much slower and 1000 steps are required for one order of magnitude drop of $\Delta Q / Q$. The reason for this convergence behavior is uncertain, but some possible reasons may include use of a nonoptimum CFL number or an improper time scaling parameter. A further study to understand this behavior is necessary. The convergence rate with preconditioning, however, is fast enough to get solutions in reasonable CPU times.

Figure 8 shows streamline and temperature isolines for $\mathrm{Ra}=10^{3}, 10^{5}$, and $10^{6}$. It is well known that solutions with the Boussinesq approximation display a full antisymmetric flowfield with respect to the center of the enclosure. The present results show a pronounced difference and the flowfield is asymmetric. At $R a=10^{3}$, the shift of center of vortex towards the cold wall and downwards to the lower wall of the enclosure is manifest. In all cases, the basic form of the flowfield is a recirculating roll. This recirculation is driven by vorticity generated by the horizontal temperature gradient $(\partial T / \partial x)$. At $\mathrm{Ra}=10^{3}, \partial T / \partial x$ is negative over the entire flowfield and a single primary clockwise rotating roll is formed. At $\mathrm{Ra}=10^{5}$ and $10^{6}$, there are two secondary rolls embedded in the single roll base flow. These secondary rolls are generated because at high Ra numbers the intense development of thermal boundary layers in the vicinity of the wall leads to the opposite sign of $\partial T / \partial x$. Also it can be noted that as Ra increases, the secondary rolls intensify and their centers move towards the side walls. The accuracy of numerical solutions is verified by comparing the Nusselt number at the left side wall with a correlation by Chenoweth and Paolucci [17] in Fig.9. Good agreement can be observed for all three cases.

\section{Summary}

Extension of the time-derivative preconditioning method to viscous flows has been considered. A previously developed preconditioning method fails in viscous flows because of nonphysical time reversal for diffusive terms. In order to circumvent the difficulty, a "viscous" set of primary dependent variables is introduced. With these primary dependent variables, the equations degenerate to the classical diffusion equations in the limit of highly diffusive flows. The proper scaling of the time derivatives is made to obtain well-conditioned eigenvalues for efficient convergence in inviscid flows. The resulting algorithm also provides a mechanism for keeping both the von Neumann number and the CFL number of order one at very viscous conditions, thereby ensuring rapid convergence at low Reynolds numbers. The quantitative effects of the new preconditioning method on the convergence of a time-marching algorithm have been investigated for several types of problems. It is shown that convergence enhancement of the preconditioning method ranges from a factor of two to several orders of magnitude.

\section{Acknowledgment}

This work is sponsored by NASA Lewis Research Center under contract NAS3-25266 with Bernard J. Blaha as project manager and by NASA Grant NAGW1356 Supplement 3.

\section{References}

1. Sovey, J. S., Hardy, T. L., and Englehart, M., "A Bibliography of Electrothermal Thruster Technology 1984," NASA TM-86998, 1986.

2. Viviand, H., "Pseudo-Unsteady Systems for Steady Inviscid Flow Calculation," Numerical Methods for the Euler Equations of Fluid Dynamics, F. Angrand et al., ed., SIAM, 1985, pp. 334-368.

3. Peyret, R. and Viviand, H., "Pseudo-Unsteady Methods for Inviscid or Viscous Flow Computations," Recent Advances in the Aerospace Sciences, C. Casci, Ed., Plenum, New York, 1985, pp. 41-71.

4. Turkel, E., "Preconditioned Methods for Solving the Incompressible and Low Speed Compressible Equations," Journal of Computational Physics, Vol. 72, Oct. 1987, pp. 277-298.

5. Storti, M. A., Baumann, C. E., and Idelsohn, S. R., "A Preconditioning Mass Matrix to Accelerate the Convergence to the Steady Euler Solutions Using 
Explicit Schemes," II WCCM, Stuttgart, August 1990.

6. Briley, W. R., McDonald, H., and Shamroth, S. J., "A Low Mach Number Euler Formulation and Application to Time-Iterative LBI Schemes," AIAA Journal, Vol. 21, No. 10, Oct. 1983, pp. 14671469.

7. Choi, D. and Merkle, C. L., "Application of TimeIterative Schemes to Incompressible Flow," AIAA Journal, Vol. 23, No. 10, Oct. 1985, pp. 15181524.

8. Choi, Y.-H., "Computation of Low Mach Number Compressible Flow," Ph. D. Thesis, The Pennsylvania State University, 1989.

9. Feng, J. and Merkle, C. L., "Evaluation of Preconditioning Methods for Time Marching System," AIAA Paper 90-0016, AIAA 28th Aerospace Sciences Meeting, Reno, Nevada, Jan. 1990.

10. Dannenhoffer, III, J. F. and Giles, M. B., "Accelerated Convergence of Euler Solutions Using Time Inclining," AIAA Journal, Vol.28, No. 8, Aug. 1990 , pp. 1457-1463.

11. Merkle, C. L. and Choi, Y.-H., "Computation of Low-Speed Compressible Flows with TimeMarching Procedures," International Journal for Numerical Methods in Engineering, Vol.25, June 1988, pp. 293-311.

12. Guerra, J. and Gustafsson, B., "A Numerical Method for Incompressible and Compressible Flow Problems with Smooth Solutions," Journal of Computational Physics, Vol.63, Apr. 1986, pp. 377-397.

13. Venkateswaran, S., Merkle, C. L., and Thynell, S. T., "An Analysis of Direct Solar Thermal Rocket Propulsion," AIAA Paper 90-0136, AIAA 28th Aerospace Sciences Meeting, Reno, Nevada, Jan. 1990.

14. Hosangadi, A., Merkle, C. L., and Turns, S. R., "Analysis of Forced Combusting Jets," AIAA Journal, Vol.28, No.8, Aug. 1990, pp. 1473-1480.

15. Withington, J. P., Shuen, J. S., and Yang, V., "A Time Accurate, Implicit Method for Chemically Reacting Flows at All Mach Numbers," AIAA Paper 91-0581, AIAA 29th Aerospace Sciences Meeting, Reno, Nevada, Jan. 1991.

16. Rehm, R. G. and Baum, H. R., "The Equations of Motion for Thermally Driven, Buoyant Flows," Journal of Research of the National Bureau of
Standards, Vol.83, No.3, May-June 1978, pp. 297308.

17. Chenoweth, D. R. and Paolucci, S., "Natural Convection in an enclosed vertical air layer with large horizontal temperature differences," Journal of Fluid Mechanics, Vol.169, 1986, pp. 173-210.

18. McMurtry, P. A., Jou, W. H., Riley, J. J., and Metcalfe, R. W., "Direct Numerical Simulations of a Reacting Mixing Layer with Chemical Heat Release," AIAA Journal, Vol.24, No.6, June 1986, pp. 962-970.

19. Douglas, J. and Gunn, J. E., "A General Formulation of Alternating Direction Methods-Part I Parabolic and Hyperbolic Problem," Numerische Mathematik, Vol.6, 1964, pp. 428-453.

20. Warming, R. F. and Beam, R. M., "On the Construction and Application of Implicit Factored Schemes for Conservation Laws," Computational Fluid Dynamics, H. B. Keller, ed., American Mathematical Society, Providence, RI, 1978, pp. 85-129.

21. Kayes, W. M. and Crawford, M. E., Convective Heat and Mass Thansfer, 2nd ed., McGraw Hill, New York, 1980.

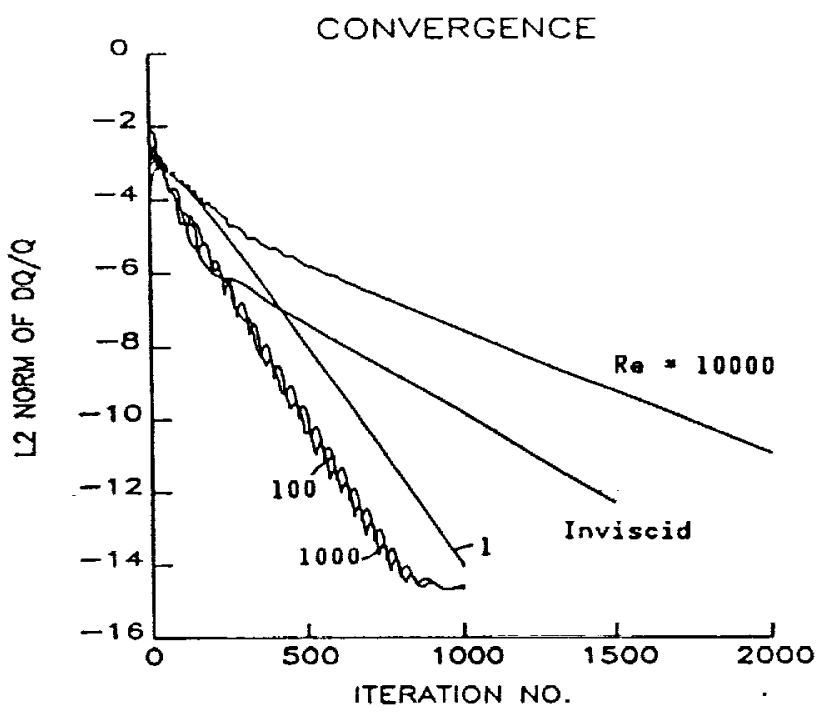

Figure 1.-Convergence rates using preconditioning method for flow past a NACAOO12 airfoil for inviscid and viscous cases, $M=10^{-4}$. For $R e=1,100,1000, C F L=6, \sigma=3$; for $R e=10000, C F L=3, k=1$ and for inviscid case $C F L=6, k=1$. 

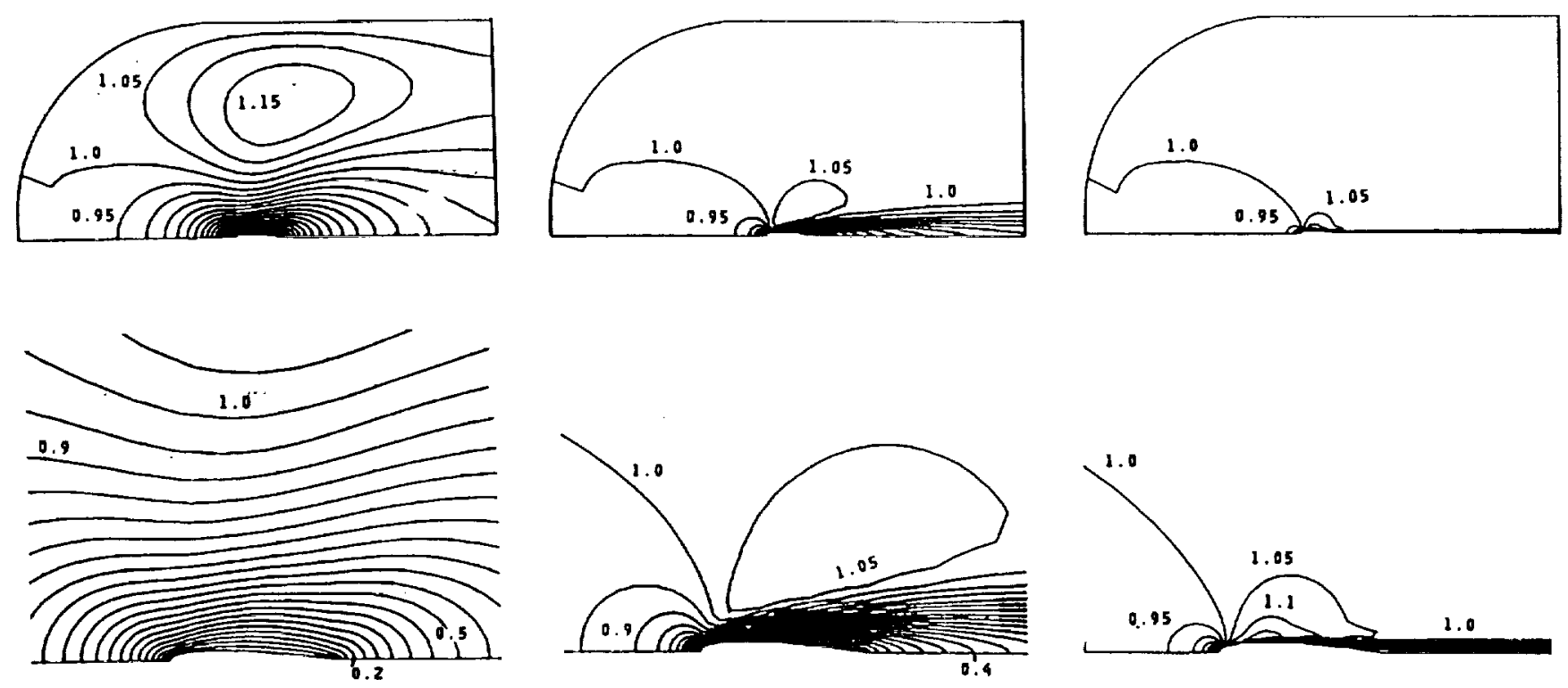

$R_{e}=1$

$R_{\mathbf{e}}=100$

$R e=10000$

Figure 2.-Velocity contour plots for viscous flow past a NACA0012 airfoil for Reynolds numbers of 1, 100, and 10000.

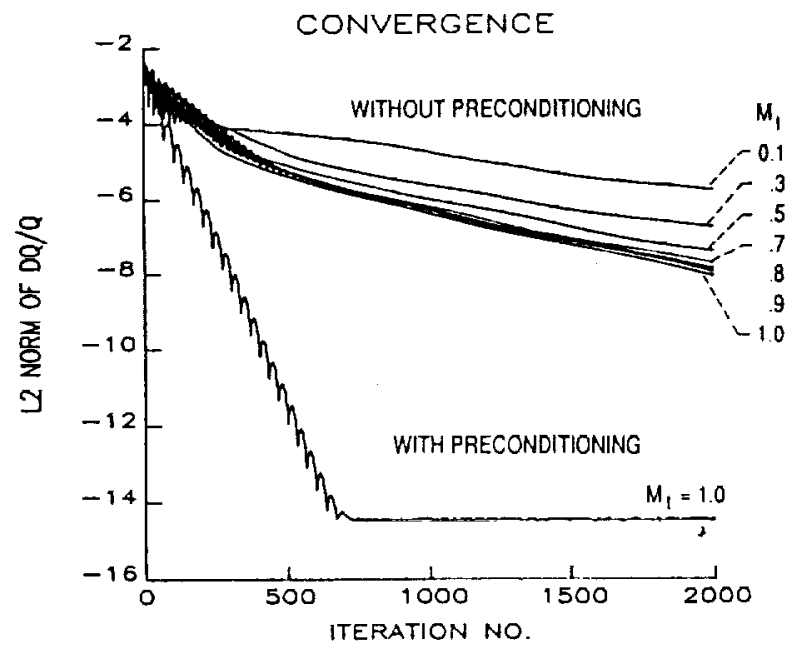

Figure 3.-Convergence rates with and without preconditioning method for inviscid flow in strongly converging-diverging nozzle $(A R=10) . M$ refers to the throat Mach number. $C F L=4$, $k=1$.

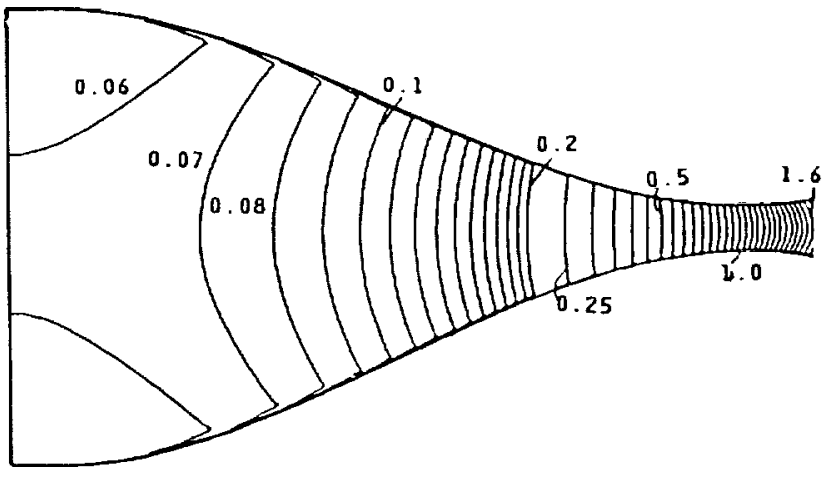

Figure 4.-Mach number contour plot in a strongly convergingdiverging nozzle $(A R=10)$. 


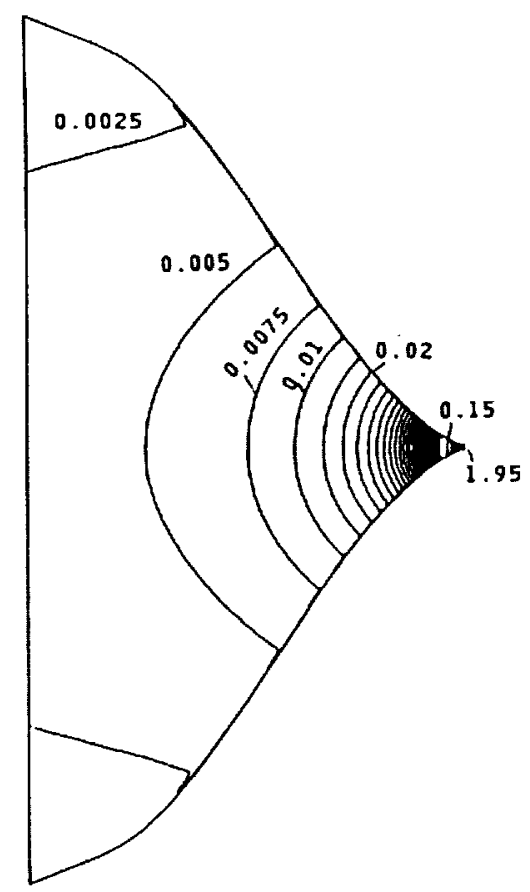

Figure 5.- Mach number contour plot in a strongly converging-diverging nozzle $(A \mathrm{~A}=200)$.

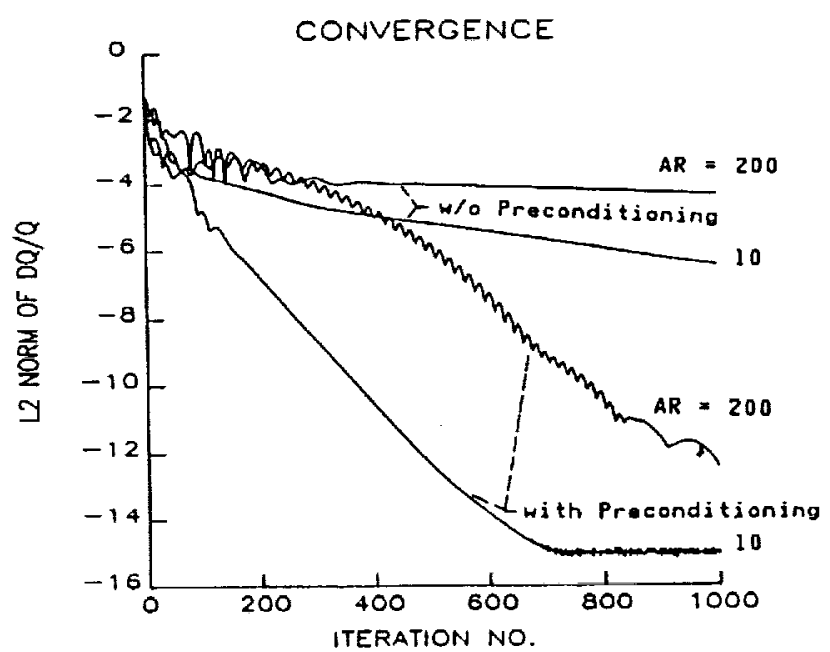

Figure 6.-Convergence rates with and without preconditioning method for viscous flows in a strongly converging-diverging nozzle $(A R=10$ and 200$)$. $C F L=7, k=1$.

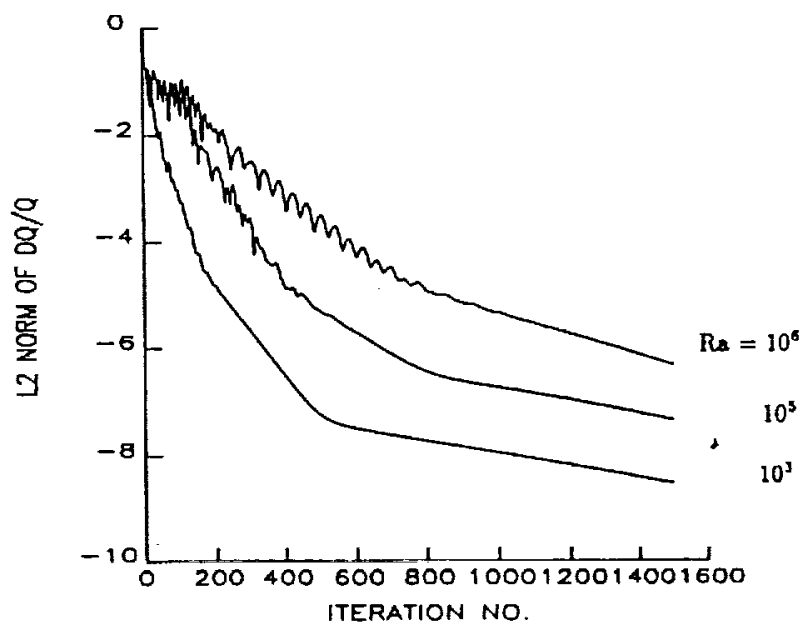

Figure 7.-Convergence rates with preconditioning method for viscous flow in a thermally driven cavity for $\mathrm{Ra}=10^{3}, 10^{5}$ and $10^{6} . \mathrm{CFL}=8, \mathrm{~K}=10$. 

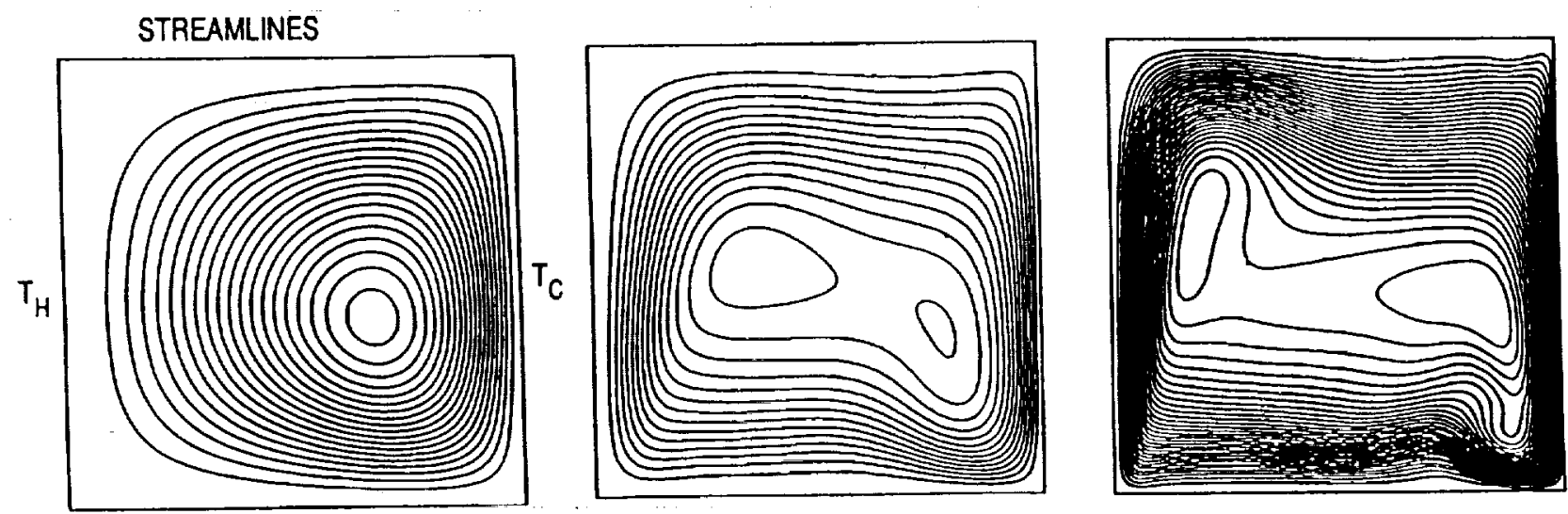

TEMPERATURE
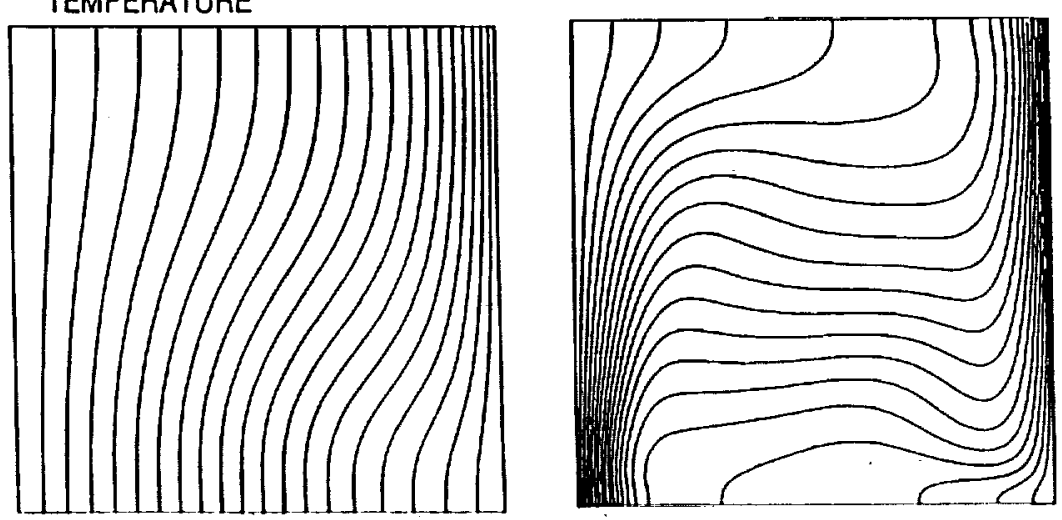

$\operatorname{Ra}=10^{3}$

$\mathrm{Ra}=10^{5}$

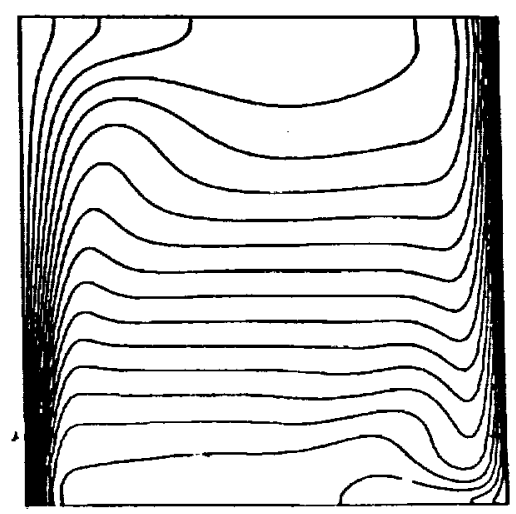

$\mathrm{Ra}=10^{\circ}$

Figure 8. Streamline and isoline temperature contours for a viscous flow in a thermally driven cavity for $\mathrm{Ra}=10^{3}, 10^{5}$ and $10^{6}$.

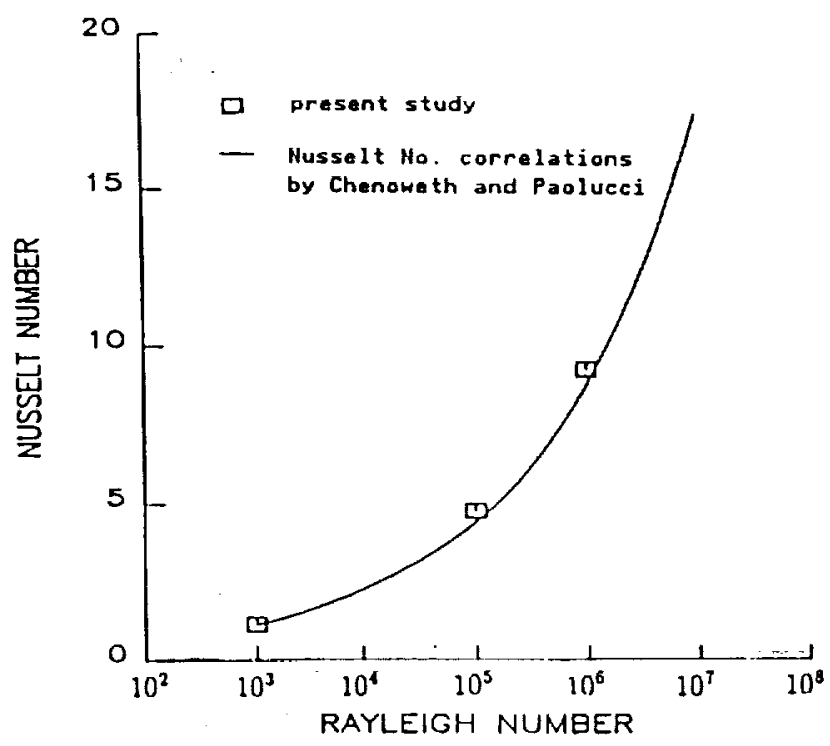

Figure 9.-Comparison of Nusselt number with a correlation by Chenoweth and Paoullicci [17]. 


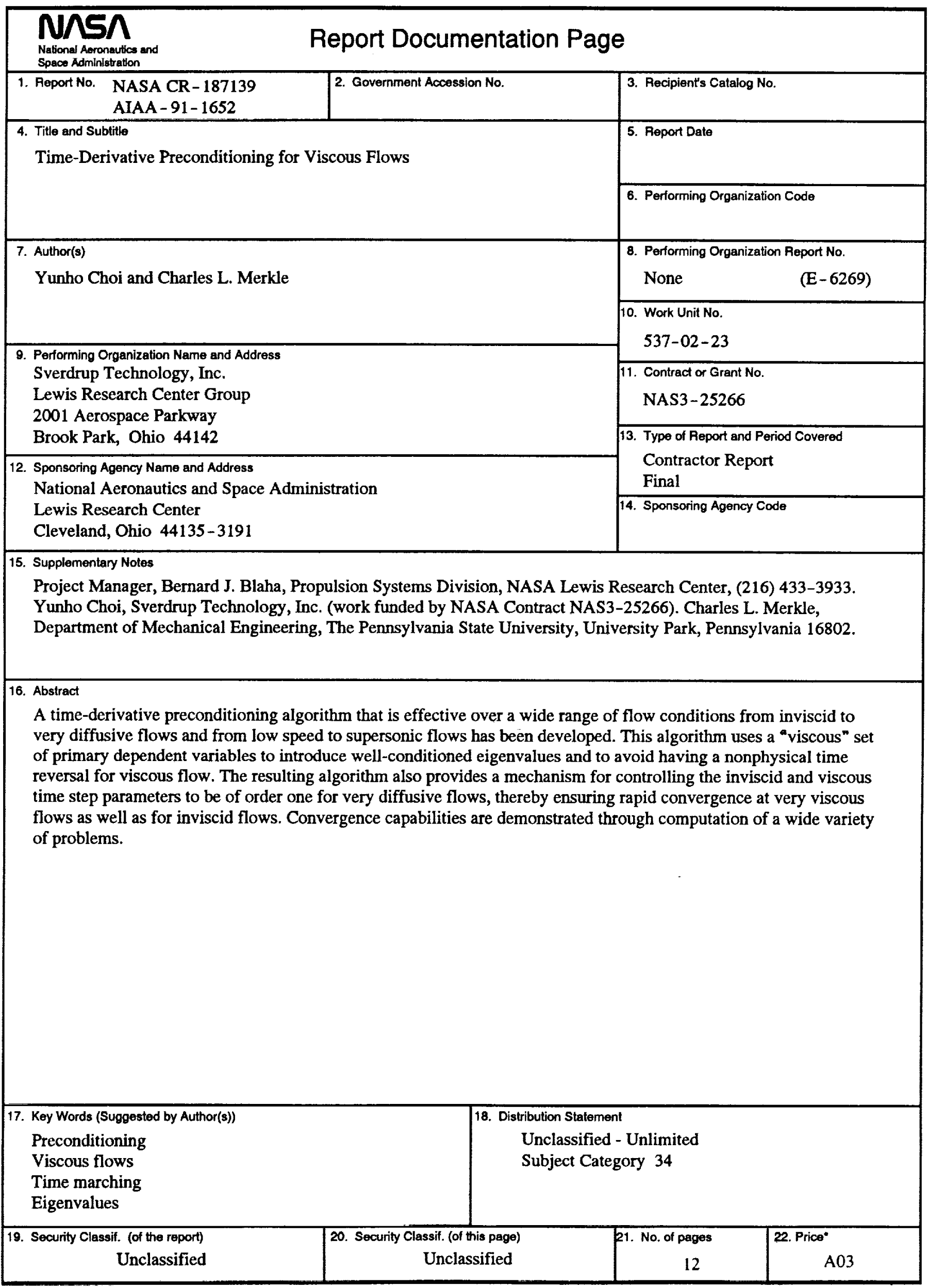

